\title{
Croix de chemin et dévotions populaires dans la Beauce
}

\section{Paul Jacob}

Volume 43, 1976

URI : https://id.erudit.org/iderudit/1007227ar

DOI : https://doi.org/10.7202/1007227ar

Aller au sommaire du numéro

Éditeur(s)

Les Éditions Historia Ecclesiæ Catholicæ Canadensis Inc.

ISSN

0318-6172 (imprimé)

1927-7067 (numérique)

Découvrir la revue

Citer cet article

Jacob, P. (1976). Croix de chemin et dévotions populaires dans la Beauce.

Sessions d'étude - Société canadienne d'histoire de l'Église catholique, 43, 15-33.

https://doi.org/10.7202/1007227ar

Tous droits réservés @ Les Éditions Historia Ecclesiæ Catholicæ Canadensis Inc., 1977
Ce document est protégé par la loi sur le droit d'auteur. L'utilisation des services d’Érudit (y compris la reproduction) est assujettie à sa politique d'utilisation que vous pouvez consulter en ligne.

https://apropos.erudit.org/fr/usagers/politique-dutilisation/ 


\title{
Croix de chemin et dévotions populaires dans la Beauce
}

\begin{abstract}
Dans son ouvrage intitulé Culte populaire des saints en Savoie, Van Gennep écrit :
\end{abstract}

Juger de la piété, et des formes de cette piété, d'une population donnée d'après seulement les textes liturgiques et leurs irradiations écrites, c'est admettre une idée non pas précisément fausse, mais insuffisante ${ }^{1 .}$

Régis Jean reconnaissait sensiblement la même chose lorsqu'il soulignait que l'étude des monographies paroissiales ne parvenait pas à nous faire connaître les dévotions d'une région déterminée. Aussi suggérait-il de mener une vaste enquête orale sur les croyances et pratiques populaires retrouvées dans les comtés de Portneuf et du Lac Saint-Jean.

C'est dans cet esprit que nous avons inscrit la modeste enquête menée récemment dans la Beauce, pour découvrir si le contenu des niches de croix de chemin pouvait être révélateur des dévotions populaires des Beaucerons. Il ne fallait pas pour autant négliger les autres sources manuscrites susceptibles de mieux nous éclairer et d'apporter une réponse plus nuancée à la vaste question qui nous était posée. Ainsi, d'une part les monographies paroissiales de quatre municipalités (Sainte-Marie, Saint-Elzéar, Saints-Anges et Saint-Séverin), d'autre part quelques manuscrits de chapelles domestiques nous ont été d'un précieux secours dans la découverte des dévotions du Beauceron.

Ces dévotions nous ont d'abord été connues sous un angle qui était loin de satisfaire nos attentes; lors d'une première entrevue avec 19 informateurs beaucerons provenant de plusieurs localités, nous nous sommes attardés exclusivement aux dévotions reliées aux croix de chemin; par la suite, nous avons choisi sept infor-

1 Arnold Van Gennep, Culte populaire des saints en Savoie, Paris, Maisonneuve et Larose, 1973, p. 158. 
mateurs provenant d'un territoire apparenté physiquement et psychologiquement; ce sont ces informateurs qui feront avant tout le sujet de notre étude, et qui symboliseront pour nous l'homme religieux de la Beauce.

L'étude des dévotions populaires soulevait par contre une difficulté majeure suggérée par le champ sémantique du vocable "populaire ». Connaître la piété et les formes de piété du peuple beauceron par leurs strictes irradiations populaires, c'est-à-dire telles qu'elles se manifestent à travers la chapelle rurale, les oratoires privés et les croix de carrefour, aurait supposé une enquête beaucoup plus vaste que la nôtre. D'un autre côté, nous ne devions pas taire la source souvent liturgique des dévotions du Beauceron; nous l'avons trouvée à l'intérieur des monographies paroissiales consultées. Le cadre de notre étude nous a fait ainsi ranger sous la rubrique "populaire " des dévotions ou des pratiques religieuses retrouvées au sein du peuple, et qui tiennent à la fois de la discipline ecclésiastique et de la coutume locale.

Malgré la densité du sujet que nous avions à cerner, il était opportun en premier lieu de dégager la perception qu'a notre informateur de la niche de croix de chemin; par la suite, nous avons jeté un rapide coup d'œil sur le répertoire des sujets de statuettes qui habitent les 17 niches visitées. Le temps fort de notre recherche consistera cependant dans l'analyse des sources consultées: monographies paroissiales et enquêtes sur le terrain.

\section{I - LA NICHE ET LA CROIX DE CHEMIN}

Les dévotions populaires, nous avons tenté de les percevoir à travers le champ visuel du modeste oratoire rural qui nous a permis d'apprivoiser l'homme religieux de la Beauce: la niche de croix de chemin. Ces deux artefacts, en l'occurrence la niche et la croix, adoptent des symboles différents aux yeux de l'informateur beauceron, à qui nous avons demandé - non sans rencontrer quelques obstacles - quelle importance il attachait à l'un et à l'autre.

La plupart de nos informateurs estiment que la niche et la croix revêtent autant d'importance l'une que l'autre. C'est ainsi que nous avons obtenu les réponses suivantes: "C'est quasiment 
toute beau " ${ }^{2}$, "sont importants les deux; ça se complète; la Sainte Famille p'is Notre Seigneur $~^{3}$, " chacun a sa signification; la croix représente le chemin de la vie; la niche, elle, représente la confiance en la Sainte Vierge $"{ }^{4}$.

Ceux qui centrent davantage leurs regards sur la croix n'oublient pas le symbole de rédemption qui y est rattaché. Nous l'avons deviné derrière les remarques plutôt évasives de deux informatrices de Saint-Joseph : " on est porté à penser à Notre-Seigneur quand on voit la croix " 5 ; " quand je passe devant la croix, je dis toujours : bénissez-moi, sainte croix (...) c'est pas nécessairement le saint qu'il y a dans la niche ${ }^{6}{ }^{6}$. Monsieur Joseph Bédard, de Sainte-Marie, affirme plus catégoriquement que «la croix est plus importante que tout le reste " 7 , mais il n'en donne pas le motif.

Bien peu nombreux sont ceux qui donnent à la niche une préséance sur la croix. Pour expliciter leur pensée, ils répondront tour à tour que la niche donne l'occasion d'avoir une dévotion particulière ${ }^{8}$ vu qu'elle constitue une décoration puisque " rien qu'une croix, c'est nu ${ }^{9}$. Monsieur Antonio Huppé, de SaintElzéar, accorde à la niche un caractère proprement sacré car, à ses yeux de chrétien, elle « remplace le tabernacle dans l'église » 10; image d'autant plus forte que l'on n'est pas sans ignorer que la croix de chemin, pour le paysan, devenait bien souvent le premier substitut de l'église.

Mais comment savoir les motifs internes qui ont incité les paysans à ajouter une niche à la croix ? Pour imiter le modèle d'une première croix, qui en comportait déjà une, nous ont répondu quelques personnes; par élément de décoration ou souci de beauté, évoqueront certains: "pour embellir la croix; avec une statue bénite en d'dans, c'est encore plus beau " ${ }^{11}$; par souci de suivre

2 Carnets d'enquête, croix 280. Inf. : A. Labbé, Saints-Anges.

3 Ibid. : croix 244. Inf. : Mme Fernand Roy, Saint-Joseph.

4 Ibid.: croix 345. Inf. : $M^{\text {me }}$ Valérien Cyr, Saint-Elzéar.

5 Carnets d'enquête, croix 236. Inf.: Mme Philippe Jacques, SaintJoseph.

6 Ibid.: croix 233. Inf. : $M^{\text {me }}$ Louis-Aimé Cliche, Saint-Joseph.

7 Ibid. : croix 348. Inf. : Joseph Bédard, Sainte-Marie.

8 Ibid. : croix 240. Inf. : Wilfrid Vachon, Tring-Jonction.

9 Ibid.: croix 300. Inf. : Alcide Ferland, Saint-Séverin.

10 Ibid. : croix 295. Inf. : Antonio Huppé, Saint-Elzéar.

11 Carnets d'enquête, croix 240. Inf. : Wilfrid Vachon, Tring-Jonction. 
la mode, nous dira $\mathbf{M}^{\text {me }}$ Arthur Gagné, de Saint-Elzéar : "parce que c'était la mode; ça s'est toujours faite de même, une croix avec la niche " ${ }^{12}$. Enfin, sept informateurs relient la présence de la niche au besoin d'exercer un culte particulier. C'est ainsi que M. Roméo Vachon, de Saint-Séverin, a construit spécifiquement la niche qui orne la croix de chemin du rang Sainte-Anne pour permettre à ses voisins de pratiquer leurs dévotions à la thaumaturge.

La niche, donc, qu'une première enquête nous fit connaître de manière superficielle. Mais qui nous a dit, à travers ces réponses à des questions souvent incomprises, qu'il fallait se rapprocher davantage du paysan et communier plus étroitement à lui pour connaître les aliments de sa vie intérieure. Qui nous a dit, surtout, qu'il fallait aller au delà de la question; et que, derrière la présence de la niche, se trouvait caché ce caractère sacré que recherche l'homme, qu'il ne peut nommer en des mots, sinon ceux de son âme.

La niche est là, attenante à la maison. C'est là qu'est stationné le sacré duquel l'homme voudra obtenir assistance et bénédiction:

Aussi n'est-il pas moins nécessaire de protéger le sacré de toute atteinte du profane ${ }^{13}$.

Ce sacré, un seul informateur nous l'a laissé entrevoir en des mots qui expriment bien ce qui devait germer en chaque paysan dans son geste d'ériger une croix de chemin et de l'orner d'une niche. "Pour rappeler la présence du Christ vivant dans le tabernacle ${ }^{14}$ : geste sacré, geste de l'homme témoin de sa foi populaire.

Il nous a donc fallu nous approcher davantage de la niche de croix de chemin, cet objet de dévotion populaire qui répond à l'énoncé de Van Gennep : «telle petite statue d'oratoire a entendu plus de prières directes que tel tableau enfumé pendu aux murs d'une cathédrale ${ }^{15}$. Psychologiquement très proche de la masse des croyants, n'est-elle pas une des multiples expressions (avec

12 Ibid. : croix 292. Inf. : Mme Arthur Gagné, Saint-Elzéar.

13 Roger CaIlloIs, L'homme et le sacré, Éditions Gallimard, 1950, p. 19.

14 Carnets d'enquête, croix 295. Inf. : M. Antonio Huppé, SaintElzéar.

15 Arnold Van GenNeP, op. cit., p. 158. 
l'oratoire domestique et la chapelle rurale) de l'imagerie populaire, pleine de naiveté et de vie intense ? En somme, la niche manifeste de l'intervention localisée de la dévotion populaire. Et elle sait nous dire ce que fut la dévotion populaire des Beaucerons en ce qu'elle demeure pour nous un signe palpable et signifiant de cette dévotion.

Notre préoccupation de connaître les dévotions du beauceron devait prendre un premier point d'appui - bien fragile - auprès des sujets actuels ${ }^{15 a}$ retrouvés à l'intérieur des niches de croix de chemin. Ce répertoire suppose, par le biais de onze statuettes de la Vierge, l'attachement religieux de la Beauce pour la mère de Jésus. Rien de bien étonnant, car c'est là une des grandes dévotions du peuple canadien-français. La Beauce ne pouvait échapper à un culte d'envergure que traduisent clairement les monographies paroissiales et l'enquête auprès des informateurs privilégiés. Nous soulignons ici, à la lumière du carnet d'enquêtes, que treize des dix-sept croix de chemin répertoriées ont été explicitement témoins des dévotions collectives du peuple à la sainte Vierge, dans le cadre bien connu des anciens mois de Marie. Cependant, il est nettement insuffisant de limiter les dévotions populaires du Beauceron à ce culte du mois de Marie, ou à des pratiques religieuses mariales qui se sont déroulées aux croix de chemin.

Attenant au culte de la Vierge se rangent d'autres dévotions bien connues et illustrées par deux statuettes de sainte Anne, deux autres de la sainte Famille et une seule du Sacré-Cœur. Il nous faut toutefois dépasser le cadre étroit de la dévotion aux croix de chemin - objet d'une première enquête - pour connaître les motifs du choix de telle statuette plutôt que de telle autre.

Ainsi, chez $M$. et $\mathbf{M}^{\text {me }}$ Roméo Vachon, de Saint-Séverin, la figurine de sainte Anne semble avoir été choisie pour commémorer le nom du rang, en l'occurrence le rang Sainte-Anne. Monsieur le curé Arsenault y est souvent venu réciter le chapelet à l'occasion de la neuvaine de sainte Anne, mais d'autres dévotions ont surgi autour de celle-ci, et qui ne se situent pas strictement dans les préoccupations de la sainte : de 1938 à 1940, par exemple, le chapelet récité à l'occasion du mois de Marie voyait s'adjoindre des

15a Car les sujets de statue se sont vu quelquefois changer au cours des années (Croix 239, 240, 280, 236, 233 et 295). 
intentions bien particulières, que $\mathbf{M}^{\mathrm{m}}$ Vachon a précisées à notre endroit : l'on priait alors "pour la paix dans le monde, pour ceux qui souffraient des horreurs de la guerre, pour que les hommes soient exempts de la guerre, pour la paix dans les foyers $" 15 \mathrm{~b}$. N'était-ce pas d'ailleurs en période de crise que M. Vachon avait fait ériger cette croix?

Pareillement, les dévotions pratiquées aux croix de chemin 244 et 293 ne rendent pas un hommage particulier à la sainte -Famille, dont la statuette orne la niche. N'ayant pu rencontrer ces informateurs qu'une seule fois, nous n'avons pu savoir comment se traduisaient leurs dévotions à la sainte Famille.

Enfin, la seule niche où nous ayions retrouvé une statue du Sacré Cour (croix 240) s'inscrit dans la même veine d'inspiration que les exemples que nous venons d'évoquer; pas de culte au SacréCœur, mais des dévotions à la Vierge de même que des dévotions privées sur lesquelles l'informatrice n'a pas voulu s'attarder.

D'autres sources allaient nous aider à clarifier le champ d'investigation qui était nôtre; la croix de chemin demeurait trop spécifique et ne nous permettait pas d'établir une corrélation assez étroite avec le saint (e) enfermé dans l'habitacle de la niche. La consultation des monographies paroissiales ainsi que la rencontre de quelques informateurs privilégiés nous a dit davantage sur la foi pratiquante du paysan, sur son besoin du religieux pour l'accompagner dans son geste quotidien.

\section{II - ANALYSE DES SOURCES}

\section{A) Monographies paroissiales}

Nos informateurs privilégiés proviennent de quatre paroisses de la Beauce: Sainte-Marie, Saint-Elzéar, Saint-Séverin et SaintsAnges. Il nous semblait plus juste d'étudier le culte religieux au sein de ce territoire en vertu du fait que les paroisses de SaintElzéar, Saint-Séverin et Saints-Anges ont été détachées, vers le milieu du dix-neuvième siècle, de la paroisse-mère de Sainte-Marie. Cette paroisse originaire - tout comme celle de Saint-Joseph -

15b Carnets d'enquêtes, croix 327. Inf. : $\mathrm{M}^{\mathrm{me}}$ Roméo Vachon, SaintSéverin. 
était disséminée, en 1831, sur un territoire très vaste $(67,332$ arpents de terre occupés) et comptait déjà une population de 5,113 âmes. Cet état de faits amène l'abbé Honorius Provost à écrire :

On conçoit aisément qu'avec une telle population (...) le service religieux incombant à la cure de Sainte-Marie était devenu irréalisable ${ }^{16}$.

Si bien que, lentement, Sainte-Marie dut connaître un processus de démembrement qui s'échelonna jusqu'à 1898, date d'érection canonique de la paroisse de l'Enfant-Jésus, et qui donna naissance à une dizaine de paroisses "dont quelque partie de territoire fut un certain temps sous sa juridiction spirituelle ${ }^{17}$.

Nous n'en pouvons pas conclure pour autant que les quatre paroisses dont nous analyserons les monographies ont été nécessairement le théâtre d'un culte identique. Cependant, il semble bien que ce processus de démembrement que connut Sainte-Marie ne l'a pas empêché de continuer à demeurer le centre névralgique des dévotions du Beauceron; l'enquête orale s'inscrit en ce sens, puisqu'elle aussi nous ramène à cette hypothèse. Une parenté physique doublée d'une parenté religieuse rapproche étroitement les paroisses de Sainte-Marie, Saint-Elzéar, Saint-Séverin et Saints-Anges.

Ainsi donc, au cours des prochaines pages, nous tenterons de faire ressortir, à la lumière des monographies paroissiales consultées, ces fragments de culte qui semblent lier le peuple beauceron dans une même ferveur religieuse.

Le culte marial est présent dans les pages de l'histoire religieuse de nos paroisses. Il prend, bien sûr, son point d'appui à Sainte-Marie de Beauce, ainsi que se plaît à l'évoquer l'abbé Provost lorsqu'il décide d'interrompre la longue litanie des dons administratifs des curés pour donner quelques annotations sur leurs préoccupations religieuses.

Monseigneur Joseph-Édouard Feuiltault, curé de 1896 à 1946, aurait été l'apôtre de la dévotion mariale à Sainte-Marie. Dès

16 Honorius Provost, Sainte-Marie de la Nouvelle-Beauce; histoire religieuse, Québec, Société historique de la Chaudière, p. 537.

17 lbid., p. 537. 
1898, en effet, l'annonce du mois de Marie «lui avait donné l'occasion de professer sa dévotion mariale. Il exhortait fortement à suivre cet exercice, à l'église, chaque soir, mais il se souciait de l'ordre: "personne dans les galeries. De la dévotion véritable, désintéressée, partant du cœur... Que ceux qui ne peuvent venir à l'église honorent la Très Sainte Vierge à leur maison. Pas de mois de Marie dans les écoles... des rassemblements qui ne sont pas des exercices spirituels (...) Quant aux oratoires, j'avoue que c'est dangereux : toujours des réunions ${ }^{18}$.

Le prélat changera son fusil d'épaule lorsque, dans les heures sombres de 1917-1918, il imposera aux institutrices de réciter le rosaire aux écoles. Il est par ailleurs étonnant de constater que $\mathbf{M}^{\mathrm{gr}}$ Feuiltault, dans sa crainte des exercices de dévotion aux oratoires, animera plus tard, pendant une dizaine d'années, les processions aux biens de la terre dont nous ferons plus longue mention au cours de ce travail.

Sous l'égide de la Vierge se place surtout, dans l'ordre des dévotions liturgiques, le rosaire, dont $\mathrm{M}^{\mathrm{gr}}$ Feuiltault propagea la dévótion au cours de son règne de curé. N'est-ce pas lui qui, le 3 octobre 1897, avait installé et béni publiquement dans l'église un groupe du Très Saint Rosaire ? Dès son arrivée à Sainte-Marie, le pasteur se chargea de présider annuellement cet exercice à la fête du Très Saint Rosaire. Il ne devint un exercice public quotidien qu'en 1918, à la suite de l'incendie du clocher de l'église. Un peu plus loin dans sa monographie, Provost fait remonter la pratique du rosaire au 17 juin 1917; elle aurait été alors inspirée par le danger des grandes inondations qui menaçaient la Beauce. Mais la vertu du rosaire s'étendait à d'autres calamités que les crues de la Chaudière. Témoin ces bribes extirpées des prônes de $\mathrm{M}^{\mathrm{gr}}$ Feuiltault : "Saint Rosaire pour la conversion des possesseurs d'alambics, ceux qui s'en servent, les partisans de la boisson sous toutes ses formes 》 et "Fréquentations trop longues. Moyen d'interrompre ces veillées: réciter le chapelet et même le Très Saint Rosaire " ${ }^{19}$. N'est-ce pas aussi une des dévotions qui puisse rapporter le plus d'indulgences?

Prenant, une fois, le Rosaire à lui seul, il additionne les indulgences partielles attachées à la récitation publique, à l'église,

$18 \quad$ Ibid., p. 186.

19 Ibid., p. $195 \mathrm{~s}$. 
et arrive au chiffre impressionnant de 373,000 jours d'indulgence ${ }^{20}$.

Soulignons d'autre part que c'est dans le cadre des grandes retraites qui eurent lieu au début de 1899 que s'organisa à SainteMarie la Ligue des Enfants de Marie; cette association pieuse, devenue classique, avait pour double but de cultiver chez ses membres la dévotion à Marie et de les inviter à se consacrer à elle.

La dévotion à la Vierge n'est pas exclusive à la paroisse de Sainte-Marie, bien que seule la monographie de Provost nous relate des détails substantiels sur ce culte. Ainsi, à Saints-Anges, l'abbé F.X. Leclerc, jadis vicaire à Sainte-Marie, prolongea la dévotion au rosaire que lui avait inculquée $\mathbf{M}^{\mathrm{gr}}$ Feuiltault. Quant à la paroisse de Saint-Séverin, elle connut également cette dévotion sous le règne du curé J.-B.-Léon Delisle (1920-1926).

Il faut supposer ici que Saint-Elzéar n'échappa point au raz-de-marée du rosaire, dont Léon XIII, en 1883, avait institué officiellement la récitation dans les églises. Selon les désirs et les intentions du Souverain Pontife, les fidèles catholiques devaient donc sanctifier les mois de mai et d'octobre "par l'assistance aux exercices publics dans les églises où ils auront lieu, par la récitation quotidienne du chapelet, par de ferventes communions ${ }^{21}$.

Le même enthousiasme se retrouve dans les pratiques de dévotion qui s'adressent au Sacré-Cœur. Ainsi, le 4 novembre 1880, « $\mathrm{M}^{\mathrm{gr}}$ A. Taschereau, archevêque de Québec, accordait une indulgence de 40 jours à qui réciterait un Notre-Père et un Ave en regardant avec foi et confiance la statue du Sacré-Cœur de Jésus, qui était dans l'église de Saint-Séverin. Et ce, autant de fois et aussi souvent qu'on remplissait les conditions requises "22. On s'étonne que l'abbé Arsenault nous fasse part, par la suite, du témoignage suivant :

Ce qu'il y avait d'excellent et de fécond, dans ces dévotions, c'est qu'on y appartenait librement, qu'on priait parce qu'on

20 Ibid., p. 187.

21 S.A., «Le mois du Saint-Rosaire, » La Semaine Religieuse de Montréal, 14e année, Vol. 28, no 13 (sept. 1896), p. 202.

22 Antonio ARSENAult, 'Centenaire de Saint-Séverin; 1872-1972, p. 59. 
voulait prier. Et si elles ont perdu tant de vogue, c'est parce qu'on a oublié que la prière est la plus grande force qui mène le monde 23 .

A Sainte-Marie, l'origine du développement de la dévotion au Sacré-Cœur et, en particulier, de l'adoration du premier vendredi de chaque mois remonte au 25 décembre 1875; c'est la date où fut fondée parmi les élèves du collège «la petite société du Divin Cœur de Jésus, par l'initiative du Frère Timothée, appuyé de l'abbé Onézime Naud ${ }^{24}$. $\mathrm{M}^{\mathrm{gr}}$ Feuiltault, pour sa part, concrétisera sa dévotion au Sacré-Cœur, recommandée dès les débuts de sa cure, par l'érection d'un monument qui fit écho à la campagne de Benoît XV; ce dernier, en une période où la guerre faisait rage en Europe, "demandait au monde des prières plus ferventes et composait lui-même une touchante supplique au Sacré-Cœur ${ }^{25}$. On n'oubliera pas, non plus, le zèle de $\mathbf{M}^{\mathbf{g r}}$ Feuiltault à distribuer chaque mois, à l'autel du Sacré-Cœur, le Messager de l'Apostolat de la Prière; c'est autour de ce dernier mouvement, organisé parallèlement à Saints-Anges, sous le curé Bourque (1883-1891), que se développèrent, à Sainte-Marie comme dans toutes les paroisses catholiques du Canada, les Ligues du Sacré-Cœur (fondées à Montréal par le Jésuite Édouard Hamon). Elles se situent dans la même veine d'inspiration que les Quarante Heures et les premiers vendredis du mois, dignes reliquats de l'apparition du Sacré-Cœur à sainte Marguerite-Marie et des promesses qui accompagnèrent cette apparition.

Aborder le culte à sainte Anne dans le cadre de ce mémoire n'est pas chose facile. D'une part, nous ne voulons pas reprendre le chapitre qu'Honorius Provost a consacré à la thaumaturge dans sa monographie; d'autre part, la densité du sujet vient s'affronter aux limites de notre étude, qui vise à circonscrire ici la présence régionaliste d'un des cultes qui ont connu le plus d'expansion dans la Beauce; le culte à sainte Anne, malgré qu'il ait connu lui aussi une tangente liturgique, en ce sens que le peuple y a participé d'une façon globale. Aussi ne nous attarderons-nous qu'à ses manifestations les plus populaires.

23 Ibid., p. 86.

24 Provost, op. cit., p. 443.

25 Ibid., p. 188. 
Ils sont nombreux, les motifs de fondation de la chapelle Sainte-Anne de Sainte-Marie; la sainte, qui a donné son nom à 72 paroisses ou missions du Canada, fut proclamée par Pie XII, en mai 1876, patronne de la province ecclésiastique de Québec. L'érection de cette chapelle nous ramène à la coutume que nos ancêtres avaient apportée de France: celle d'ériger des chapelles ou des calvaires au bord des routes, soit pour accomplir un veu, soit pour perpétuer le souvenir d'un miracle.

Des critères plus prochains sont proposés par Honorius Provost. $\mathrm{Au}$ sommet de ceux-ci, l'auteur place «la volonté expresse de l'autorité religieuse, l'idée d'un culte régional à Sainte Anne et d'un sanctuaire commun pour toutes les paroisses de la NouvelleBeauce ${ }^{26}$. L'érection de la chapelle, tout en satisfaisant la piété des habitants - la famille Taschereau en premier - supprimait en même temps la distance presque infranchissable que devaient parcourir les Beaucerons pour se rendre à Beaupré; elle prolongeait ainsi le culte de Beaupré. Enfin, les fondateurs de la chapelle voulaient, par ce geste, être préservés des ravages des inondations.

Autour des trois chapelles qui se sont remplacées successivement en 1778,1832 et 1891 gravitent des dévotions qui allèrent toujours en s'accroissant. Dès 1778, la ferveur et le nombre de pèlerins dépassaient les prévisions. C'était peut-être du fait que la modeste chapelle avait hérité d'une statue en bois de sainte Anne réputée miraculeuse ! A partir de 1780, la dévotion à sainte Anne connut un élan considérable: cent messes par année, venue de prédicateurs chevronnés, pèlerinages organisés venant des paroisses extérieures (avec présence du curé), affluence des pèlerins surtout à l'occasion de la fête de sainte Anne. D'ailleurs, le curé Villade ne confia-t-il pas à son évêque les propos suivants ?

La chapelle de Sainte-Anne, qui est le lieu de dévotion de toute la Beauce, y attire une infinité de personnes que je puis dire d'une piété et d'une vertu exemplaires. La plupart voudrait y apporter des rosaires et des scapulaires pour les faire bénir après qu'elles auraient entendu la Sainte Messe et communié 27 .

L'ampleur de la dévotion fut atténuée par une ordonnance de $\mathbf{M}^{\mathrm{gr}}$ Plessis, en 1810, qui faisait suite aux désordres publics

26 Ibid., p. 235.

27 Ibid., p. 247. 
(boisson) engendrés par les pèlerinages. Mais elle reprit de plus bel avec la construction de la deuxième chapelle (1828-1832) et "les indulgences et privilèges spirituels qui vinrent l'enrichir graduellement ${ }^{28}$. Notons en particulier l'acquisition, auprès du pape Grégoire XVI, d'un crucifix de bois noir, avec un Christ en argent et un reliquaire à la base duquel les pèlerins pouvaient désormais vénérer des parcelles de vêtements ou des ossements sacrés; la venue de la relique de sainte Anne, "détachée d'une parcelle d'un petit doigt de sainte Anne " ainsi que «le privilège de la sainte réserve " ${ }^{29}$ augmentèrent également le prestige de l'oratoire, confirmé par déjà deux miracles.

Si les débuts de la troisième chapelle (1891) ne nous intéressent pas particulièrement, ici, la période dite contemporaine voit apparaître des manifestations de dévotion populaire qui ne manquent pas d'éclat. Ce sont, d'une part, les processions annuelles pour les biens de la terre et, d'autre part, des processions en chaloupes sur la Chaudière; alors que les premières sont le fruit de l'imagination de $\mathbf{M}^{\mathrm{gr}}$ Feuiltaut, les secondes furent inspirées par un de ses vicaires, l'abbé Victorin Germain.

Les processions annuelles des voitures, qui s'ébauchaient à partir de la chapelle Sainte-Anne, furent inaugurées le 12 juin 1911 et durèrent officiellement jusqu'à la fin du règne de $\mathbf{M}^{\mathrm{gr}}$ Feuiltault; elles étaient dirigées contre tous les fléaux susceptibles d'affecter les cultivateurs, leurs troupeaux, leurs récoltes. Voici comment l'abbé Provost décrit ces processions de haute couleur :

Le curé faisait une forte recommandation au prône et c'était dès le lundi matin que l'événement se déroulait. Après une messe chantée à la chapelle Sainte-Anne, pour les biens de la terre, le curé paraissait sur le perron pour réciter les litanies et autres prières du Rituel. Puis tout le monde montait dans les voitures à chevaux (...) et la procession s'ébranlait. La voiture de tête portait une croix de bois noir; le curé venait ensuite, dans sa voiture, bénissant les champs et les maisons le long du parcours; puis tout le monde suivait, sans ordre convenu, les occupants de chaque voiture récitant le chapelet (...). Le parcours était toujours le même, le seul vraiment commode : montée par la route du moulin Chassé, traversée par le centre $\mathrm{du}$ rang Saint-Gabriel et descente par la route Carter. Il y eut,

28 Ibid., p. 253.

29 Ibid., p. 254. 
dans les débuts, une grande quantité de voitures à cette procession originale. À partir du 6 juillet 1925, la chapelle Turcotte se trouvant construite, on arrêta désormais à mi-chemin du parcours, pour $y$ chanter une autre grand-messe... après quoi chacun regagnait son logis 30 .

Mais les processions en chaloupes, commencées en 1928 et poursuivies jusqu'en 1936, témoignent davantage de la dévotion à sainte Anne. Inspirée à l'abbé Germain par une vague tradition locale, rapportant que plusieurs pèlerins voyageaient jadis par eau, ici tout aussi bien qu'à Sainte-Anne de Beaupré, elles attirèrent en peu de temps des foules estimées jusqu'à 20,000 personnes :

Le nombre des barques - il y en eut jusqu'à 63 - et le luxe de leurs décorations s'accrurent avec les années; on avait toujours l'ambition de faire mieux (...) chaque association paroissiale, chaque industrie rivalisait avec les autres participants, et la dépense était considérable ${ }^{31}$.

La popularité de cette procession est toute indiquée par la présence du Cardinal Villeneuve aux cérémonies de 1933, 1934 et 1935 :

On avait alors la chaloupe cardinalice, qui n'était pas la moins brillante, évidemment; une autre voiturait la chorale, une autre la statue de Sainte Anne; c'étaient plutôt des pontons flottants supportés par deux ou plusieurs chaloupes réunies 32 .

Somme toute, la chapelle de sainte Anne, jusqu'à ces dernières années, fut le théâtre de plusieurs démonstrations à caractère mi-religieux, mi-pompeux (l'orgueil des Beaucerons y serait-il pour quelque chose ?) Indiscutablement, les paroisses de Saint-Elzéar, Saint-Séverin et Saints-Anges y ont participé. Si l'auteur de la monographie de Saint-Séverin les a ignorées, celui de Saints-Anges nous a rappelé, lors d'une entrevue, que les paroissiens de cette localité ont participé, dès les débuts de Saints-Anges (vers 1875) à ces pèlerinages qui se sont faits processionnellement, en voitures, jusqu'à l'arrivée des automobiles. Rien n'empêchait les gens de se rendre en pèlerinage à Sainte-Anne de Beaupré; ce type de pèlerinage, sous le curé Bourque (1896-1891), était astreint à des règles

\footnotetext{
$30 \quad$ Ibid., p. 193.

31 Ibid., pp. 275-276.

32 Ibid., p. 275.
} 
très sévères : abstention de nourriture pour pouvoir communier, voyage en steamboat, récitation du chapelet en passant à toutes les églises, parler modérément ou prier, se mettre en procession quatre par quatre.

Le dévotion à sainte Anne connaît enfin, à Saint-Séverin, une manifestation que nous n'avons pas voulue passer sous silence :

Le dimanche, 26 juillet 1906, après la vénération de la relique de la Bonne Sainte Anne, il y a bénédiction des chapelets, des scapulaires et des médailles. Si vous n'avez pas de scapulaire sur votre poitrine, parce que vous n'y croyez pas, seriez-vous surpris qu'il vous arrive des accidents de toutes sortes ? ${ }^{33}$

Mais les grandes dévotions à la Vierge, au Sacré-Cœur et à sainte Anne voisinent d'autres dévotions que soulignent tantôt les monographies paroissiales, tantôt les curés actuels des localités étudiées. En voici le court bilan.

Si la dévotion à la sainte Famille n'est pas illustrée concrètement dans les documents qui ont été mis à notre disposition, elle apparaît quand même dans l'association harmonieuse des paroisses de Sainte-Marie, Saint-Joseph et l'Enfant-Jésus; le dernier nom de cette trilogie avait été suggéré par le Cardinal Taschereau qui, en 1891 et 1892 , avait «publié des mandements pour la restauration de cette dévotion qui lui était chère " ${ }^{34}$. Sous $\mathbf{M}^{\mathrm{gr}}$ Feuiltault, également, la dévotion à saint François-Xavier fut propagée pendant quelques années sous la forme d'une neuvaine de la grâce. La période choisie annuellement pour cette neuvaine était fondue avec les Quarante Heures et comportait un " concours de dévotions visant à sanctifier le temps du carnaval $»^{35}$.

Ce n'est là qu'une maigre représentation, compte tenu du fait que les dévotions des Beaucerons nous ont semblé esquissées faiblement dans les monographies paroissiales; les auteurs ne s'y attardent que passagèrement, préférant faire revivre l'histoire de leur paroisse sous des angles avant tout historiques. Ainsi, l'abbé Provost a beau dire que "la paroisse de Sainte-Marie est réputée pour sa floraison étonnante d'oratoires publics ou privés » ${ }^{36}$; il faut

33 Antonio ARSenault, op. cit., p. 74.

34 P'Rovost, op. cit., p. 559.

35 Ibid., p. 187.

36 Ibid., p. 191. 
dépasser les lignes pour connaître ce qui, par exemple, se cache derrière les quatre chapelles de dévotion qui font partie de l'histoire religieuse de Sainte-Marie.

\section{B) Enquêtes sur le terrain}

Un second type de documents, celui de l'enquête orale sur le terrain, constituera enfin le point capital de notre étude, puisqu'il est relié aux grandes dévotions que dégagent les monographies paroissiales et qu'expriment, en l'occurrence chez nos informateurs privilégiés, les statuettes retrouvées dans les niches de croix de chemin. L'enquête menée auprès de ces informateurs nous permet d'établir unc première constatation : la dévotion suggérée par la statue de niche est pratiquée, à des degrés plus ou moins forts, par le propriétaire de la croix de chemin. Mais cette dévotion est entourée d'autres cultes à divers saints ou d'autres pratiques religieuses; il serait hasardeux d'affirmer qu'elle prime sur les autres dévotions. Néanmoins, la présence de la statue est l'indice sûr d'autres dévotions qui accompagnent le culte au saint enfermé dans l'habitacle de la niche.

La Vierge qui orne la niche de croix de chemin, chez M. Alcide Ferland, de Saint-Séverin ${ }^{37}$, ne semble pas un simple fétiche; elle est véritablement objet de culte, et comme la statuette de la Vierge placée devant l'horloge de la cuisine, elle a entendu plusieurs prières, en particulier le chapelet quotidien (chapelet sur rebord d'une fenêtre); c'est en elle que $M$. Ferland a placé sa confiance, et c'est elle qui le protège. Notre informateur l'a souvent priée à l'occasion des rassemblements du mois de Marie à la croix du chemin. Sa dévotion à la Vierge trouve une double origine; d'une part, il nous a avoué être né pendant le mois de Marie; d'autre part, à l'âge de 10 ans, il fut "reçu du scapulaire " de la sainte Vierge qu'il porta jusqu'à il y a quarante ans pour le remplacer par des médailles à l'effigie de sainte Anne et du Sacré-Cœur. Chez ce premier informateur, le culte à la Vierge n'est pas exclusif; il s'entoure de dévotions à sainte Anne, à sainte Thérèse, à saint Joseph et à la sainte Famille. Sa femme soulignera, à ce propos, une remarque plus éloquente que toute dissertation: "Pour moé, i's ont toutes la même force parce qu'i' est fort comme un ours ».

37 Croix 300. Inf. : A. Ferland, Saint-Séverin. 
Dans le rang Sainte-Anne, à Saint-Séverin, s'élève une autre croix de chemin ${ }^{38}$; dans la niche, une statuette de sainte Anne que la famille Vachon et les gens du rang honorent à l'occasion de la neuvaine de sainte Anne présidée chaque année par le curé Arsenault. Membre des femmes chrétiennes, $\mathbf{M}^{\mathrm{me}}$ Vachon se consacre chaque mois à sainte Anne, récite souvent son petit chapelet de sainte Anne et porte, avec son scapulaire, une médaille à l'effigie de la thaumaturge. Elle reconnait cependant avoir autant confiance à la sainte Vierge : " J'ai confiance aux deux, dit-elle, autant l'une comme l'autre ». Sa dévotion au Sacré-Cœur rejoint aussi celles que nous avons esquissées dans les monographies paroissiales; elle a ceci de particulier qu'elle s'exprime à travers le chapelet de la Sainte Plaie.

$\mathbf{M}^{\text {me }}$ Vachon a longtemps récité ce chapelet avec son mari, mais elle l'a discontinué depuis que quelqu'un aurait dit «qu'on prenait une dévotion un peu trop en récitant ce chapelet». $M^{\text {lle }}$ Valéda Richard, de la même localité, abonde en ce sens lorsqu'elle nous déclare que l'évêque ou le pape aurait suggéré « de réciter le chapelet individuellement parce qu'en groupe il y avait trop de grâces ». Au-delà de toutes les dévotions pratiquées par $\mathbf{M}^{\mathrm{me}}$ Roméo Vachon prime cependant la croix : "Quand on prie, c'est par la croix qu'on se sauve. Si ç'avait pas été d'la croix (...) NotreSeigneur n'a pas établi le Notre-Père pour rien. Le Saint Sacrifice de la messe, c'est si important ».

A l'instar de cette informatrice, $\mathbf{M}^{\mathrm{me}}$ Irenée Groleau, de Saints-Anges, a canalisé ses dévotions autour de la statue de la Vierge qui repose dans la niche de leur croix de chemin ${ }^{39}$. $\AA$ tous les jours, en plus de réciter avec ses enfants le chapelet en famille, elle les confie à la Vierge " pour pas avoir de misère avec eux autres ". $\mathbf{M}^{\text {me }}$ Groleau, à côté de ce culte principal, se confie également à sainte Anne : "J'la prie pareil ", nous a-t-elle dit. Aussi, chaque année, fait-elle sa neuvaine de sainte Anne en plus de se rendre en pèlerinage à la chapelle Sainte-Anne de Sainte-Marie. La foi de cette famille trouve une dernière manifestation assez caractéristique : dans deux imposants poulaillers attenant à la maison, se trouvent deux statues du Sacré-Cœur: "on les a mis là dans l'poulailler pa'ce que ça allait mal une secousse; on aurait mis des statues de la Sainte Vierge, mais on n'en trouvait pas ».

38 Croix 327. Inf. : Mme Roméo Vachon, Saint-Séverin.

39 Croix 282. Inf. : $M^{\text {me }}$ Irenée Groleau, Saints-Anges. 
Il semblerait que ce soit la sainte Vierge qui ait exaucé les souhaits des paysans du rang Saint-Thomas, de Saint-Elzéar; ceux-ci avaient promis d'ériger une croix et d'y bâtir une niche à la Vierge si le gouvernement ouvrait un chemin aux habitants du rang. L'enquête menée auprès de la famille Cyr n'atteste que de quelques dévotions populaires à cette croix de chemin 40; vers les années 1935, une maîtresse et ses élèves venaient y réciter le chapelet. Les gardiens de la croix, $\mathbf{M}$. et $\mathbf{M}^{\text {me }}$ Valérien Cyr, ont cependant continué leurs dévotions à la sainte Vierge, à qui ils se recommandent chaque jour : "on invoque un peu le Sacré-Cœur, souligne $\mathbf{M}^{\mathrm{me}} \mathrm{Cyr}$; mais le Sacré-Cœur et la Sainte Vierge, ça va toute ensemble $»$.

Nous avons trouvé moins de tiédeur chez $\mathbf{M}$. et $\mathbf{M}^{\mathrm{me}}$ Antonio Huppé, de Saint-Elzéar; selon eux, en effet, " on prie jamais trop ». Et les deux statuettes qui se sont remplacées dans la niche, depuis que la croix de chemin ${ }^{41}$ se trouve sur leur emplacement, sont en relation très étroite avec les pratiques religieuses de ces paysans. La sainte Vierge et sainte Anne sont au centre des dévotions de ce couple. Ainsi, $M^{\text {me }}$ Huppé nous a avoué qu'à chacun de ses accouchements, elle gardait dans ses mains une médaille de sainte Anne; depuis les premières années de son mariage, aussi, elle récite chaque jour son petit chapelet de sainte Anne («si je l'ai pas, me semble que j'dormirai pas $\|$ ); pour sa part, son mari accorde sa préférence à la sainte Vierge. Il tient sa dévotion du curé Alphonse Labbé, qui fut jadis le vicaire de $\mathbf{M}^{\mathrm{gr}}$ Feuiltault, à Sainte-Marie, de 1924 à 1944.

Chez $M$. et $\mathbf{M}^{\text {me }}$ Joseph Bédard, de Sainte-Marie, nous avons retrouvé également une grande ferveur religieuse qui nous fut connue par le biais de la belle croix de chemin ${ }^{42}$ qui domine leur propriété. À l'intérieur de la niche y figure une statue de l'Immaculée Conception auprès de laquelle, de 1933 à 1973, se centralisaient les dévotions au mois de Marie qui réunissaient une trentaine d'adultes et d'enfants du voisinage. $\mathbf{M}^{\mathrm{me}}$ Bédard avoue d'ailleurs que c'est la sainte Vierge qui gardait naguère ses enfants quand elle allait aux champs. Elle n'a pas perdu sa foi en Marie, puisqu'elle récite chaque soir son chapelet avec son mari: "Moi,

40 Croix 345. Inf.: Mme Valérien Cyr, Saint-Elzéar.

41 Croix 295. Inf. : M. et $M^{\text {me }}$ Antonio Huppé, Saint-Elzéar.

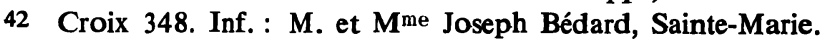


je l'continue, çartain. J'écoute pas l'monde... Aujourd'hui i's le r'nouv'ent. " L'Immaculée Conception, dans les dévotions de ce couple âgé, semble bien insuffisante. Ainsi, l'étroite proximité avec la chapelle Sainte-Anne de Sainte-Marie fait en sorte que la patronne du Québec a trouvé en $\mathbf{M}^{\mathrm{m}}$ Bédard une digne militante; pendant une vingtaine d'années, $\mathbf{M}^{\text {me }}$ Bédard a fait, chaque soir de juillet, la neuvaine de sainte Anne à la chapelle de Sainte-Marie (messe, sermon, communion, vénération de la relique): "ça nous a jamais raculés durant les foins», a-t-elle précisé. Aujourd'hui, elle se contente de réciter son petit chapelet de sainte Anne (un Pater et trois dizaines de cinq Ave chacune entrecoupées par deux "Gloire soit au Père »).

\section{CONCLUSION}

Le contenu des niches de croix de chemin est-il révélateur des dévotions populaires du Beauceron? Nos informateurs privilégiés nous ont répondu par l'affirmative; et bien que leur faible nombre ne nous permette pas d'apporter une conclusion définitive, il a permis au moins de dégager la constante mise en lumière dans la dernière partie du travail: les statuettes de niche nous mènent sûrement vers les dévotions du peuple. Là où la niche a été posée, des dévotions multiples se sont exercées et s'exercent encore. Non pas seulement à l'endroit du sujet représenté dans la niche - ce sujet faisant figure de symbole religieux - mais à l'adresse des saints promus par le clergé.

À travers le biais des monographies paroissiales, le clergé nous est apparu dans ce travail comme la grande ligne de force qui atteint le peuple et qui monopolise les cultes aux grands saints. Et si, par exemple, nous nous sommes longuement attardés sur l'ardeur religieuse de $M^{\mathrm{gr}}$ Feuiltault, c'est qu'elle nous disait concrètement que le clergé a eu longtemps le privilège de prêter au peuple ses dévotions, tout en lui indiquant la manière de les pratiquer. Aussi s'est-il retrouvé dans la confusion la plus complète lorsque la bourrasque religieuse de la dernière décennie l'a forcé à jouer un second rôle dans la vie de ses paroissiens. L'abbé Provost en témoigne d'ailleurs assez bien quand il écrit :

Ces manifestations de piété populaire sont pour ainsi dire inconcevables aujourd'hui (...) Qu'avons-nous pour les remplacer? La science, les inventions, la mécanique ont pris le 
pas sur les sacramentaux et on oublie de bénir les insecticides, les tracteurs (...); un grand nombre ne font plus attention aux grains bénits des rogatiens, aux litanies qu'on récitait avant de jeter en terre la première poignée de semence.

Nous avons observé d'autre part que le clergé a très souvent rejoint le peuple dans les dévotions qui sortent du lieu intimiste de l'église. Les processions aux biens de la terre, jadis menées à Sainte-Marie de Beauce, voyaient se joindre le pasteur; pareillement, l'animation des prêtres aux réunions du mois de Marie, à la croix du chemin, revêtait un caractère parfois plus populaire que liturgique. Ce curé, qui attirait des enfants à la croix de chemin, en leur distribuant des bonbons et en farcissant le religieux de feux d'artifice, ne laisserait-il pas supposer que le clergé devient peuple et que le liturgique se confond très vite au populaire ?

En définitive, la présente étude nous a mis en présence d'un sujet dont les racines étaient multiples. La niche de croix de chemin nous a fait plonger au sein de l'homme religieux de la Beauce, que nous ne connaissons encore que d'une manière trop fragmentaire.

Paul JACOB,

École des gradués

(Arts et Traditions populaires)

Université Laval. 